\title{
Myocardial Motion Analysis and Visualization from Echocardiograms
}

\author{
Michael Sühling ${ }^{a}$, Muthuvel Arigovindan ${ }^{a}$, Christian Jansen $^{b}$, Patrick Hunziker ${ }^{b}$, and \\ Michael Unser $^{a}$ \\ ${ }^{a}$ Swiss Federal Institute of Technology Lausanne, CH-1015 Lausanne VD, Switzerland \\ ${ }^{b}$ University Hospital Basel, CH-4031 Basel, Switzerland
}

\begin{abstract}
We present a new framework to estimate and visualize heart motion from echocardiograms. For velocity estimation, we have developed a novel multiresolution optical flow algorithm. In order to account for typical heart motions like contraction/expansion and shear, we use a local affine model for the velocity in space and time. The motion parameters are estimated in the least-squares sense inside a sliding spatio-temporal window.

The estimated velocity field is used to track a region of interest which is represented by spline curves. In each frame, a set of sample points on the curves is displaced according to the estimated motion field. The contour in the subsequent frame is obtained by a least-squares spline fit to the displaced sample points. This ensures robustness of the contour tracking. From the estimated velocity, we compute a radial velocity field with respect to a reference point. Inside the time-varying region of interest, the radial velocity is color-coded and superimposed on the original image sequence in a semi-transparent fashion. In contrast to conventional Tissue Doppler methods, this approach is independent of the incident angle of the ultrasound beam.

The motion analysis and visualization provides an objective and robust method for the detection and quantification of myocardial malfunctioning. Promising results are obtained from synthetic and clinical echocardiographic sequences.
\end{abstract}

Keywords: Motion estimation, motion visualization, time-varying deformable model, echocardiography

\section{INTRODUCTION}

Echocardiography is one of the most widely used diagnostic techniques to evaluate the heart function of patients with known or suspected heart disease. It is non-invasive, relatively non-expensive and fairly simple to use. The analysis of ventricular wall motion, in particular, allows to assess the extent of myocardial ischemia and infarction. ${ }^{1,2}$ In clinical practice, the detection of wall motion abnormalities is usually performed by visual inspection or manual measurements. This can be very time consuming and allows only an observer dependent diagnosis. Automating the analysis of echocardiographic images is desirable but also challenging because of the low image quality and the high amount of speckle noise. In this paper, we propose a framework for a robust, automated and quantitative analysis of echocardiographic sequences. We make two key contributions:

Motion Estimation: We propose a novel optical flow algorithm to estimate ventricular wall motion from echocardiographic sequences. In order to account for typical heart motions like contraction/expansion and shear, we use a local affine model for the velocity in space and time. The motion parameters are estimated in the least-squares sense inside a sliding spatio-temporal window. Robustness is achieved by a coarse-to-fine multi-scale strategy.

Motion Visualization: The radial component of the velocity with respect to a reference point is then visualized inside a time-varying region of interest (ROI). The ROI is defined by a set of spline curves which are tracked in time by using the estimated velocity fields. In each frame, a set of sample points on the spline curves is displaced according to the motion field and the contour in the subsequent frame is obtained by a leastsquares B-spline fit to the displaced sample points. Inside the time-varying region of interest, the radial

Correspondence: michael.suehling@epfl.ch, http://bigwww.epfl.ch/ 
velocity is color-coded and superimposed on the original image sequence in a semi-transparent fashion. The motion trajectory of the ROI-centroid, the principal axes of inertia and the area-size of the ROI are also displayed.

The paper is organized as follows. We describe the motion estimation algorithm in some detail in Section 2. The ROI-tracking and motion visualization is described in Section 3. We then validate the algorithm in Section 4 by applying it to synthetic and clinical image sequences.

\section{MOTION ESTIMATION}

Several methods have been proposed to quantify heart motion from two-dimensional (2D) echocardiograms. Special attention has been paid to optical flow methods which have led to promising results. ${ }^{3}$ Let $I(x, y, t)$ denote the intensity of pixels at location $\mathbf{r}=(x, y)$ and time $t$ in an image sequence. Gradient-based optical flow estimation relies on the assumption that the intensity of a particular point in a moving pattern does not change with time. The constant intensity assumption can be expressed as $^{4}$

$$
I_{x}(\mathbf{r}, t) u(\mathbf{r}, t)+I_{y}(\mathbf{r}, t) v(\mathbf{r}, t)+I_{t}(\mathbf{r}, t)=0,
$$

where $I_{x}, I_{y}$ and $I_{t}$ denote the spatial and temporal derivatives of the image intensity. The velocities $u$ and $v$ are, respectively, the $x$ - and $y$-components of the optical flow we wish to estimate. Since (1) is a single equation in two unknowns, $u$ and $v$, it cannot be solved uniquely without introducing additional constraints.

\subsection{Local Affine Velocity in Space-Time}

A popular optical flow algorithm is the Lucas-Kanade method, ${ }^{5}$ which estimates the motion locally, assuming that it is constant within a local window. To account for typical heart motions, such as expansion, contraction, and shear, we extend this approach to a local, spatial-affine model for the motion. Additionally, we also use a linear model for the velocity along the time direction. This allows to capture local accelerations in time much better than a locally constant model. Another advantage is that we can base our estimation on multiple frames around a given time point; this is much more robust than using only two frames as many classical optical flow methods do. Let $\mathbf{r}_{0}=\left(x_{0}, y_{0}, t_{0}\right)$ denote the center of a small spatio-temporal image region $\Omega$. Inside this local region, the spatio-temporal-affine model is defined as

$$
\left(\begin{array}{l}
u(x, y, t) \\
v(x, y, t)
\end{array}\right)=\left(\begin{array}{l}
u_{0} \\
v_{0}
\end{array}\right)+\left(\begin{array}{lll}
u_{x} & u_{y} & u_{t} \\
v_{x} & v_{y} & v_{t}
\end{array}\right)\left(\begin{array}{c}
x-x_{0} \\
y-y_{0} \\
t-t_{0}
\end{array}\right) .
$$

The parameters $u_{0}$ and $v_{0}$ correspond to the velocity at the center point $\mathbf{r}_{0}$ and $u_{x}, u_{y}, u_{t}, v_{x}, v_{y}$, and $v_{t}$ are the first order spatial and temporal derivatives of $u$ and $v$, respectively.

We estimate the local motion components by minimizing the weighted least-squares criterion

$$
\int_{\mathbb{R}^{3}} w\left(x-x_{0}, y-y_{0}, t-t_{0}\right)\left(I_{x} u+I_{y} v+I_{t}\right)^{2} d x d y d t .
$$

The symmetric window function $w$ gives more weight to constraints at the center of the local spatio-temporal region than to those at the periphery.

A very well suited window function is $w(x, y, t)=\beta^{n}(x) \beta^{n}(y) \beta^{n}(t)$, where $\beta^{n}$ is the symmetrical B-spline of degree $n \in \mathbb{N}^{6}$ B-splines rapidly converge to Gaussians when their degree increases which ensures isotropy of the window in multiple dimensions. Additionally, the B-splines satisfy a two-scale equation which allows for an efficient computation of B-spline-weighted inner products at dyadic scales by using a wavelet-like algorithm (cf. Section 2.2).

By differentiating (3) with respect to each of the eight unknown parameters, we obtain a symmetric linear $(8 \times 8)$-system $\mathbf{A} \mathbf{v}=\mathbf{b}$ in terms of local moments of the spatial and temporal derivatives of $I$. This local linear system is set up and solved for each spatio-temporal position of the sliding window. 


\subsection{Coarse-To-Fine Multi-Scale Strategy}

It is obviously difficult to estimate large motions at fine scales. A way around this problem is to apply a coarse-to-fine strategy. This is realized by dilating and shifting the window function in space by powers of two:

$$
w_{j}\left(x-x_{0}, y-y_{0}, t-t_{0}\right)=w\left(\frac{x-2^{j} x_{0}}{2^{j}}, \frac{y-2^{j} y_{0}}{2^{j}}, t-t_{0}\right) .
$$

The integer $j$ denotes the spatial scale. As shown in Fig. 1, the motion vectors are transferred from the coarser to the next finer resolution level as initial estimates and are then refined if they do not already exceed a scaledependent size. For each local estimate, we compute a confidence measure which is based on the magnitude of the residual in (3). A local estimate is refined only if its confidence measure is larger than the corresponding one at the next coarser scale. Furthermore, a local solution is regarded as not admissible if the linear system is either ill-conditioned or if the length of the estimated central motion vector exceeds some scale-dependent limit. Finally, a motion estimate is set to zero if the local mean of the time derivative at the given location is below

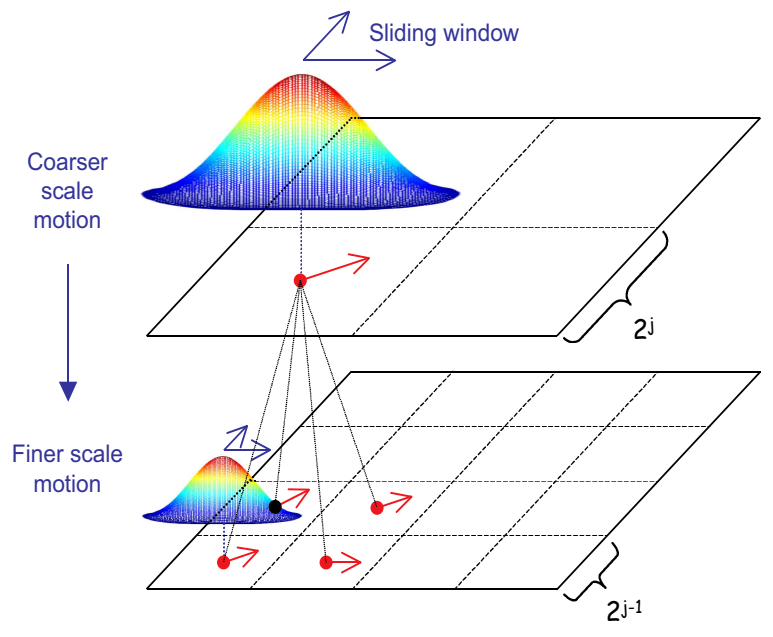

Figure 1. Coarse-to-fine multi-scale strategy in space.

a pre-defined noise level. From the discrete output, we fit a spatio-temporal spline model to obtain a global, continuous representation of the velocity field.

\section{ROI-TRACKING AND MOTION VISUALIZATION}

From the estimated velocity model, we extract and visualize radial velocity information inside a time-varying deformable region of interest (ROI). For each frame, the ROI is defined by a set of closed spline curves (typically, the inside and outside of the myocardium). For a given time $t$, each spline curve is parameterized as $\mathbf{r}_{t}(\tau)=$ $\left(x_{t}(\tau), y_{t}(\tau)\right)$ in terms of the variable $\tau$. In this paper, we represent $\mathbf{r}_{t}(\tau)$ as a linear combination of B-spline basis functions ${ }^{6}$

$$
\mathbf{r}_{t}(\tau)=\sum_{l=-\infty}^{\infty} \mathbf{c}_{t}(l) \beta^{n}\left(\frac{\tau}{m}-l\right) .
$$

The integer $n$ denotes the degree of the B-spline and $\mathbf{c}_{t}(l)$ denotes a sequence of vector spline coefficients given by $\mathbf{c}_{t}(l)=\left(c_{t}(l), d_{t}(l)\right)$. The basis functions are dilated and shifted by some integer $m$ meaning that spline knots on the curve are located at distance $m$. Since the curves are closed, the sequence of spline coefficients $\mathbf{c}_{t}$ is periodic with some period length $K$. To track a curve in time, we compute a series of sample points $\mathbf{r}_{t}(k)$, $k=0, \ldots, m K-1$, at integer distance on the curve. For these sample points, we compute the displacement from the continuous spline representation of the velocity field. By adding the displacements to the current position of the sample points, we obtain their position

$$
\hat{\mathbf{r}}_{t+1}(k)=\mathbf{r}_{t}(k)+\left(u\left(\mathbf{r}_{t}(k), t\right), v\left(\mathbf{r}_{t}(k), t\right)\right)
$$




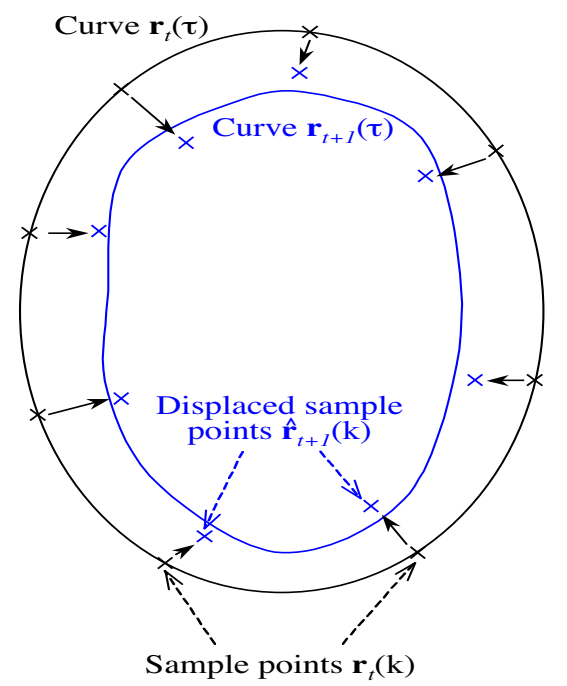

Figure 2. ROI-tracking by least-squares B-spline fitting.

in the next frame $t+1$ as shown in Fig. 2. In order to increase the robustness of the tracking process to noise, we approximate the $m K$ displaced sample points by the spline model (5) that has $K$ degrees of freedom, i.e., the spline coefficients $\mathbf{c}_{t+1}(l), l=0, \ldots, K-1$. These spline coefficients are determined by minimizing the least-squares approximation error

$$
\varepsilon^{2}=\sum_{k=0}^{m K-1}\left\|\mathbf{r}_{t+1}(k)-\hat{\mathbf{r}}_{t+1}(k)\right\|_{l_{2}}^{2} .
$$

The choice of the parameter $m$ determines the trade-off between closeness to the sample points and smoothness of the spline curve. An efficient filter-based approach to compute the spline coefficients is described in Unser et al.. ${ }^{7,8}$ The set of obtained spline curves then defines the ROI at time $t+1$. The tracking process is initialized by placing landmarks on the first frame of the image sequence to obtain the initial spline curves. The corresponding spline coefficients $\mathbf{c}_{0}(l)$ in $(5)$ are determined by B-spline interpolation of the landmarks $(m=1)$.

Having computed the ROI for each frame, we compute the centroids (centers of gravity), the areas, and the principal axes of inertia of the ROIs. These parameters can be computed exactly from the spline representation of the contours. ${ }^{9} \quad$ The velocity field of each frame is then projected onto the centroid of a particular frame (end-diastolic or end-systolic) to obtain radial velocity fields. Optionally, the velocity can also be projected onto the floating centroid of the ROI. In this case, the global translational motion of the ventricle is compensated by subtracting the velocity of the floating centroid from the estimated overall velocity. The choice of the optimal centroid (fixed vs. floating) depends on the conditions under which the heart is imaged. Under conditions where excessive translation of the heart occurs, such as might be the case after cardiac surgery, a floating centroid approach would be more appropriate.

Inside the time-varying ROI, we color-code the radial velocity using a similar color map as in Tissue Doppler Imaging (TDI). The color-coded velocity is then superimposed on the underlying image sequence in a semitransparent fashion. The motion trajectory of the centroids, the principal axes of inertia and area-size of the ROIs are also displayed. The color display helps to quantify the degree of myocardial wall excursion. The principal axes of inertia allow to identify the extend of ventricular rotation (heart-twisting).

\section{NUMERICAL RESULTS}

\subsection{Application to Synthetic Data}

We have tested our method on synthetic image sequences simulating an apical short axis view of a heart. The expansion/contraction motion of the myocardium was modeled such that its area remains constant. This results 
in a radial velocity field with a magnitude decreasing with the distance from the center. This kind of motion reflects the wall-thinning and thickening of a beating heart during diastole and systole. Additionally to the myocardial excursion, the heart model is translating towards the upper right direction to simulate an underlying rigid motion. The heart tissue is assumed to be a collection of point-scatterers with random echogenicity. The echographic signal is obtained by a convolution of the warped point-scatterer images with a point spread function (PSF). For the PSF, we have used a cosine modulated Gaussian envelope. The resulting B-mode images are given by the envelope computation of the echographic signal.

The applied motion field was then estimated by the algorithm from the simulated B-mode images. For radial motion visualization, the estimated velocity was projected onto the floating centroid of the ROI to compensate for the underlying translational motion. One frame of the synthetic sequence, the estimated velocity field and the color-coded radial velocity during diastole are shown in Fig. 3. The ROI clearly tracks the myocardium and

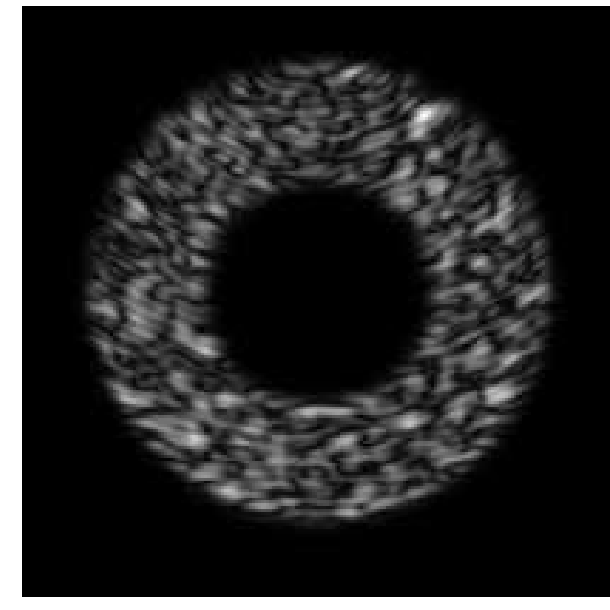

(a) Frame of synthetic sequence during diastole

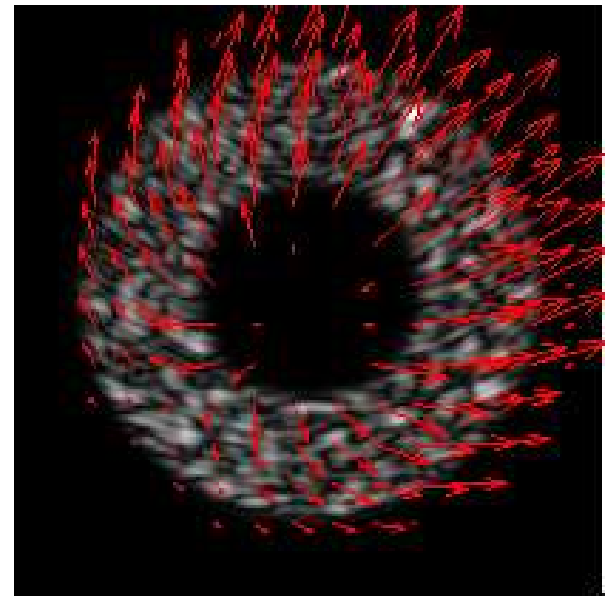

(b) Estimated velocity field

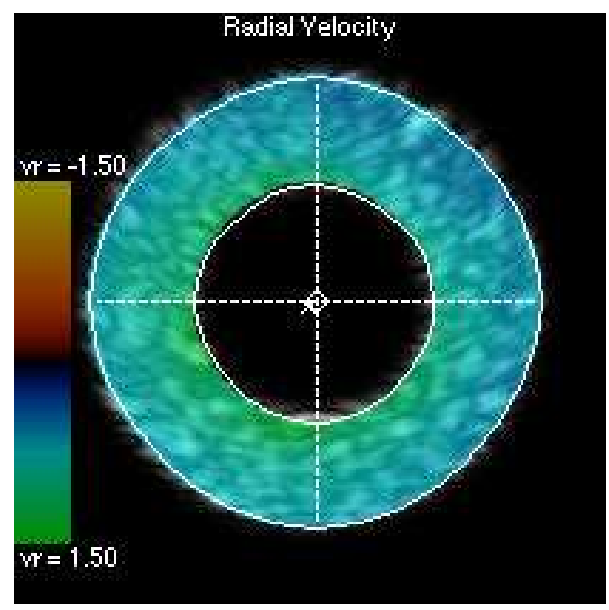

(c) Superimposed color-coded radial velocity, centroid and principal axes of inertia of the ROI

Figure 3. Results obtained from a synthetic sequence. 
the radial myocardial velocity gradient due to wall thinning is well reflected by the colors.

The error between the estimated velocity $\widehat{\mathbf{v}}$ and the exact velocity $\mathbf{v}$ is evaluated by the angular error measure

$$
\theta=\arccos \frac{\langle\mathbf{v}, \widehat{\mathbf{v}}\rangle}{\|\mathbf{v}\|_{l_{2}}\|\widehat{\mathbf{v}}\|_{l_{2}}}
$$

In this particular experiment, the maximum velocity was 2.0 pixels per frame and the average angular error over all pixels in the image sequence was $3.18 \pm 3.63$ degree.

\subsection{Application to Clinical Data}

The usefulness and efficiency of the method for detecting myocardial malfunctioning is also demonstrated by applying the method to clinical echocardiographic sequences. Fig. 4 shows one frame of a long axis view TEEechocardiogram during systole and the corresponding motion estimation results. Here, the velocity was projected onto a fixed reference point that is given by the ROI-centroid at end-diastole. The ROI clearly follows the movement of the myocardium and the colors reflect its degree of expansion and contraction. Although only partially imaged, the dyskinesia (expansion during systole) in the anterior apical segment of the myocardium (upper right segment) is clearly indicated by the green-blue color and the displayed motion field.

\section{CONCLUSIONS}

We have presented a novel framework to estimate myocardial velocity from echocardiograms and to visualize radial velocity within a time-varying deformable ROI. In contrast to conventional Tissue Doppler methods, this approach is independent of the incident angle of the ultrasound beam. In comparison to active contour approaches, which track myocardial borders, we obtain myocardial motion information inside the complete ROI. The method is accurate and robust due to a multiresolution strategy for motion estimation and the use of spline models for the velocity and the ROI-contours. The method allows to objectively detect and quantify myocardial malfunctioning and looks promising for clinical applications.

\section{ACKNOWLEDGMENTS}

This work was supported in part by the Swiss National Science Foundation under grant 3200-059517.99 and the Swiss Heart Foundation. 


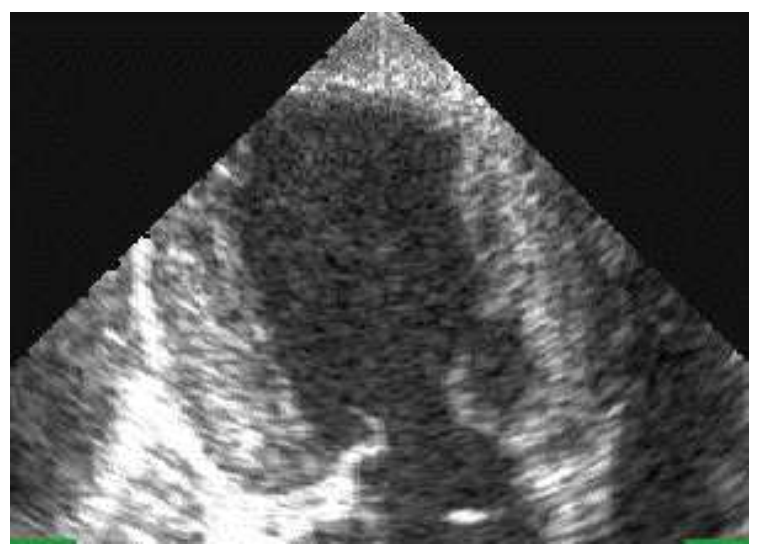

(a) Frame of sequence during systole

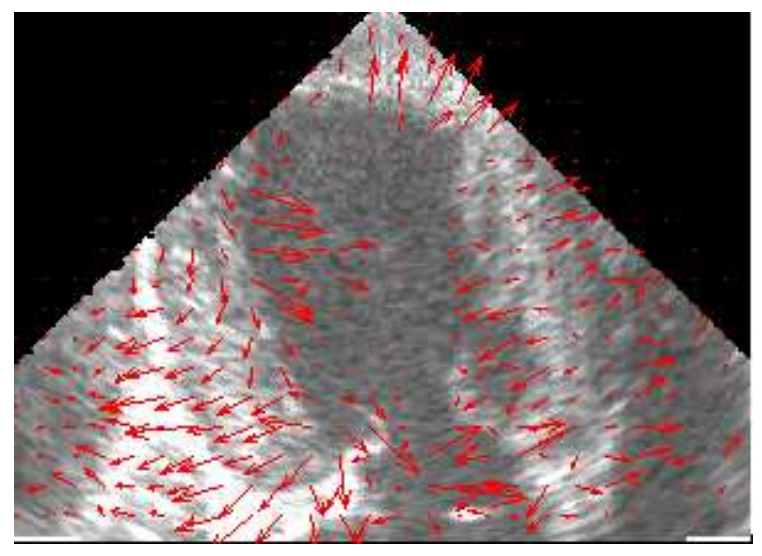

(b) Estimated velocity field

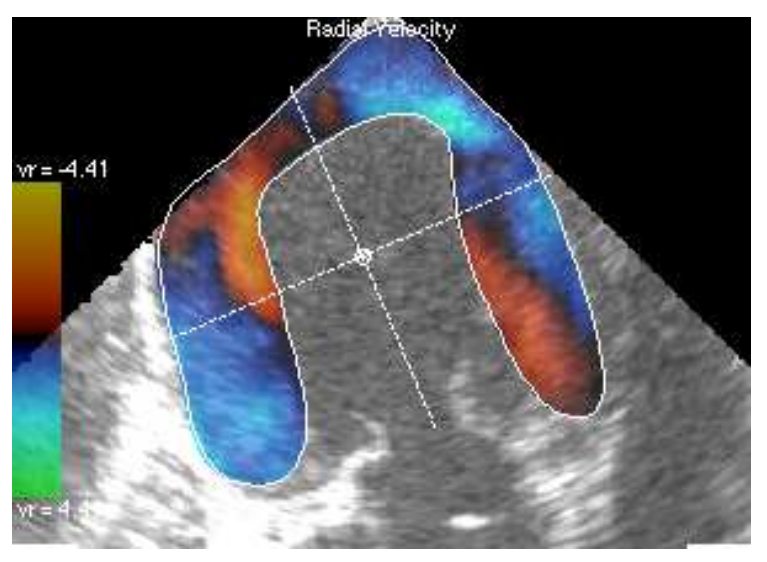

(c) Superimposed color-coded radial velocity, centroid and principal axes of inertia of the ROI

Figure 4. Results obtained from clinical data. 


\section{REFERENCES}

1. P. R. Hunziker, M. H. Picard, N. Jander, M. Scherrer-Crosbie, M. Pfisterer, and P. T. Buser, "Regional wall motion assessment in stress echocardiography by tissue doppler bull's-eyes.," Journal of the American Society of Echocardiography 12, pp. 196-202, March 1999.

2. X. Papademetris and J. S. Duncan, Handbook of Medical Imaging, vol. 2, ch. 12: Cardiac Image Analysis: Motion and Deformation. SPIE PRESS, 2000.

3. P. Baraldi, A. Sarti, C. Lamberti, A. Prandini, and F. Sgallari, "Evaluation of differential optical flow techniques on synthesized echo images," IEEE Transactions on Biomedical Engineering 43, pp. 259-272, March 1996.

4. B. Horn and B. Schunk, "Determining optical flow," Artificial Intelligence 17, pp. 185-203, 1981.

5. B. Lucas and T. Kanade, "An iterative image restoration technique with an application to stereo vision," Proceedings of the DARPA IU Workshop, pp. 121-130, 1981.

6. M. Unser, "Splines: A perfect fit for signal and image processing," IEEE Signal Processing Magazine 16, pp. 22-38, November 1999.

7. M. Unser, A. Aldroubi, and M. Eden, "B-Spline signal processing: Part I-Theory," IEEE Transactions on Signal Processing 41, pp. 821-833, February 1993.

8. M. Unser, A. Aldroubi, and M. Eden, "B-Spline signal processing: Part II-Efficient design and applications," IEEE Transactions on Signal Processing 41, pp. 834-848, February 1993.

9. M. Jacob, T. Blu, and M. Unser, "An exact method for computing the area moments of wavelet and spline curves," IEEE Transactions on Pattern Analysis and Machine Intelligence 23, pp. 633-642, June 2001. 